\title{
Visceral Afferents and Metabolic Function
}

\author{
A. Niijima \\ Department of Physiology, Niigata University School of Medicine, Niigata City, Japan
}

\begin{abstract}
Summary. The effect of glucose, 2 DG, CCK and serotonin on the firing rate of the vagal hepatic afferents and vagal pancreatic afferents was studied in rabbits, guinea pigs and rats. The hepatic and pancreatic afferents showed a decrease in activity following administration of glucose and an increase in activity after the injection of $2 \mathrm{DG}$. These afferent elements may have a mechanism to respond to blood glucose similar to that of lateral hypothalamic neurons. The results also indicate that CCK and serotonin showed the opposite effects in the vagal hepatic afferents and in the pancreatic efferents. Decrease in the discharge rate in the vagal hepatic afferents due to CCK or serotonin may cause a suppression of food intake behavior. Increase in the discharge rate in the vagal pancreatic afferents resulting from CCK or serotonin or insulin suggests the existence of a nervous information system for these substances in the circulating blood in the pancreas.
\end{abstract}

Key words: Glucose, 2 DG, CCK, serotonin, vagal hepatic afferents, vagal pancreatic afferents, insulin

It is known that blood glucose concentration is one of the important factors controlling appetite and feeding behavior. Anand [1], Oomura [18, 19] and other workers proved the existence of glucose-sensitive nerve cells in the hypothalamic region by means of electrophysiological techniques. It might be expected that these glucose-sensitive nerve cells play a role as controllers of feeding behavior as well as regulators of the rate of secretion of insulin, glucagon and catecholamines through the autonomic nervous system, to guarantee a reasonable utilization of glucose by the body and brain cells.

Russek [21] suggested the existence of additional glucoreceptors in the liver by means of behavioral studies. He suggested that these receptors monitor the glucose levels of portal venous blood through the vagus nerve and play an important role in the regulation of food intake behavior. Novin [17] has also pointed out the importance of signals originating from the visceral organs such as the stomach, duodenum and liver in the control of food intake. It has been estimated that $90 \%$ of the abdominal vagus consists $87 \%$ afferent fibers.

Recently, Mei [12] reported the existence of glucoreceptors in the wall of the duodenum and intestine. Following the intraduodenal infusion of glucose, he observed a rapid increase of afferent activity in the nodose ganglion of the vagus nerve by means of extracellular glass microelectrodes. It was reported that the stimulating effect is mainly specific for glucose. This study suggests the existence of glucoreceptors in other visceral organs such as the liver and pancreas.

The possibility that cholecystokinin (CCK), one of the gut hormones, acts as a "satiety hormone" has been proposed by several workers [2]. Serotonin ( $5 \mathrm{HT}$ ) has also long been known to be contained in the enterochromaffin (EC) cells dispersed in the gut. There has been a report that the intraventricular administration of serotonin caused a decrease of food intake. This observation raises the possibility that serotonin liberated from the gut to the portal venous blood causes a suppression of food intake through hepatic vagal afferents.

This paper reports the effects of glucose, CCK and serotonin on the firing rate of vagal hepatic afferents and vagal pancreatic afferents. The effect of insulin, 2 deoxyglucose ( 2 DG) and mannose on the activity of pancreatic afferents was also observed.

\section{Materials and Methods}

Adult rabbits, guinea pigs and rats of both sexes were used for recording afferent activity. Discharges were recorded from finely dissected afferent nerve filaments in the hepatic or in the celiac 
branches of the vagus nerve innervating the pancreas. The discharge was picked up by a pair of silver-silver chloride electrodes and amplified by means of a condenser-coupled amplifier, monitored by an oscilloscope and stored on magnetic tape. All analyses of nervous activity were performed after conversion of raw data to standard pulses by a window discriminator, which separated discharge from background noise. The standard pulses were then fed to a rate meter with a reset time of five sec for studying the time course of discharge rate. The discharge rate was displayed on a polygraph record along with the signals indicating injections.

\section{Results and Discussion}

\section{Studies on Vagal Hepatic Afferents}

Effect of Glucose. As mentioned in the introduction, the existence of glucoreceptors in the liver was reported by Russek [21]. Electrophysiological observations of these afferents were made in perfused liver preparations in the guinea pig. Afferent discharges in the hepatic branch of the vagus nerve showed a decrease in the firing rate following administration of glucose through the portal vein to the liver [13]. In the next step of these studies, experiments were conducted in guinea pigs in situ [14].

A gradual decrease of the discharge rates of hepatic afferents was observed, following an intraduodenal infusion of $5 \%$ glucose $(5 \mathrm{ml})$. It was accompanied by a gradual increase of glucose content in the portal venous blood. These observations demonstrated that an increase of glucose concentration in portal venous blood results in a decrease of the rate of signalling from the liver to the central nervous system, perhaps to the hypothalamic region, through the vagus nerve. The suppression of food intake following intra-portal or intraperitoneal administration of glucose $[21,22]$ can be explained in this light. Contrary to the effect of glucose metabolism, infusion of $2 \mathrm{DG}$, a metabolic inhibitor of glucose into the portal vein caused an increase of the vagal afferent discharge rate.

It was also confirmed that after an intraportal infusion of $15 \mu \mathrm{g}$ of ouabain, an injection of d-glucose caused no change in firing rate. The decrease in the firing rate of the hepatic afferents caused by glucose seems to be due to the activation of the energydependent sodium pump, since the reaction was effectively blocked by a cardiac glycoside such as ouabain. Oomura [20] found that the inhibitory effect of glucose on the activity of glucose sensitive neurons in the lateral hypothalamic area is also due to this mechanism.

Reflex Effects from Glucose-Sensitive Afferents. To study the reflex effect of these glucose-sensitive afferents in the liver on the activity of adrenal efferents and pancreatic efferents in the vagus nerve, we performed a number of experiments in rabbits and guinea pigs [15].
For the experiments in rabbits, a catheter was inserted into the portal vein and another catheter was placed in the left jugular vein for glucose infusion. The mean discharge rates of pancreatic and adrenal efferents were compared before and after infusion of glucose. To calculate the mean discharge rate, the number of spikes in a five-sec period was averaged over $50 \mathrm{sec}$ in the adrenal nerve and over $100 \mathrm{sec}$ in the pancreatic branch of the vagus nerve. The results show that i.v. infusion of glucose $(25 \mathrm{mg} / \mathrm{kg})$ caused no significant change in the mean discharge rate of the adrenal nerve or the pancreatic branch of the vagus nerve. However, intra-portal infusion of the same amount of glucose resulted in a significant decrease of the mean discharge rate in the adrenal nerve $(p<0.01)$. The intraportal infusion of glucose also caused a significant increase in the discharge rate of the pancreatic branch of the vagus nerve $(0.01<\mathrm{p}<0.02)$.

These results indicate that an increase of glucose concentration in the portal venous blood causes a reflex inhibition of adrenal nerve activity and reflex activation of pancreatic nerve firing. The reflex change in activities of these two nerves may cause a change in the rates of secretion of adrenaline, noradrenaline and insulin. Presumably this would cause a decrease in adrenaline and noradrenaline secretion and an increase in insulin secretion. This study suggests that glucose-sensitive hepatic afferents work as an afferent limb and vagal pancreatic efferents and adrenal efferents work as part of the efferent limbs of this reflex, and that the higher reflex centers may be in the hypothalamic region.

In the next step of the experiments, the effect of an intraportal infusion of a small amount of glucose on the firing rate of pancreatic and adrenal efferents was compared before and after the section of the hepatic branch of the vagus nerve. Guinea pigs were used for the experiments. Before nerve section, intraportal infusion of glucose $(25 \mathrm{mg} / \mathrm{kg})$ caused an increase in the firing rate of pancreatic efferents. However, after nerve sectioning, the same dose of glucose caused no significant change in the firing rate in the same nerve filament. Before the nerve sectioning, intraportal glucose also caused a decrease in the firing rate of adrenal efferents. However, after the section, the same amount of glucose caused no change in the firing rate in the adrenal nerve filament. These results indicate that the change in firing rate in pancreatic and adrenal efferents following the intraportal infusion of glucose is at least partly due to the change in the rate of nerve signals from vagal hepatic afferents, although a contribution of glucose sensitive splanchnic afferents cannot be eliminated.

In the next experiments, the effect of repetitive electrical stimulation of the hepatic branch of the vagus nerve on the firing rate of vagal pancreatic 
efferents and adrenal efferents was studied. Guinea pigs were used for these experiments. Square-wave electrical pulses of $0.1 \mathrm{msec}$ duration $(100 \mathrm{~Hz}, 3$ volts) were applied for $1 \mathrm{~min}$ to the proximal stump of the hepatic branch of the vagus nerve through a pair of silver wire electrodes which were connected through an isolation unit to a stimulator.

The repetitive stimulation of the hepatic branch of the vagus nerve caused a long-lasting decrease in firing rate of vagal pancreatic efferents and a longlasting increase in discharge rate of adrenal efferents. One min. of stimulation was usually followed by a change in the firing rate in these nerves which lasted longer than $10 \mathrm{~min}$. It was observed in the experiments on glucose-sensitive vagal hepatic afferents that a decrease in glucose content in the portal venous blood caused an increase in the firing rate of vagal hepatic afferents. From this observation it can be postulated that the frequent electrical stimulation of the hepatic branch of the vagus nerve is a reasonable simulation of a decrease of glucose content in the portal venous blood.

All of the above mentioned experiments suggest that an increase in glucose content in the portal venous blood reflexly increases insulin secretion and decreases adrenaline and noradrenaline secretion. They also suggest that a decrease in glucose levels in the portal vein promptly increases production of adrenaline and noradrenaline and inhibits insulin production as a reflex change. Perphaps glucose-sensitive afferents in the liver serve as monitors of portal blood glucose and can trigger an anticipatory response for the regulation of systemic blood glucose levels. The reflex effects of these hepatic afferents on the rate of secretion of insulin, adrenaline, noradrenaline and systemic blood glucose levels remain to be studied.

The sectioning of the hepatic branch of the vagus nerve blocks the effect of an intraportal infusion of glucose, suggesting the pathway of glucose-sensitive afferents in the liver is via the vagus nerve.

Effect of Cholecystokinin (CCK). Following the findings that $\mathrm{CCK}$ induced behavior characteristic of satiety in rats [2] and that the i.v. infusion of CCK decreases food intake in man [24], sheep [3], rabbits [10], and monkeys [9], Lorenz [11] suggested that the satiety effect of CCK is physiological. To study the effect of CCK on hepatic afferents, a small catheter was inserted into the portal vein of a rat or guinea pig. Before use, CCK (CCK-8, a gift of Dr. Squibb of to $\mathrm{S}$. C. Woods) was dissolved in isotonic saline ( $\mathrm{pH}$ 7.8). Following injection of CCK at doses of $100 \mathrm{pg}$, $1 \mathrm{ng}$ or $10 \mathrm{ng}$ in $0.1 \mathrm{ml}$ saline, a decrease in firing rate was observed. The decrease was more than $50 \%$ in some cases. The suppressive effect of CCK usually lasted fairly long; and in some cases, at 1.5 hour after
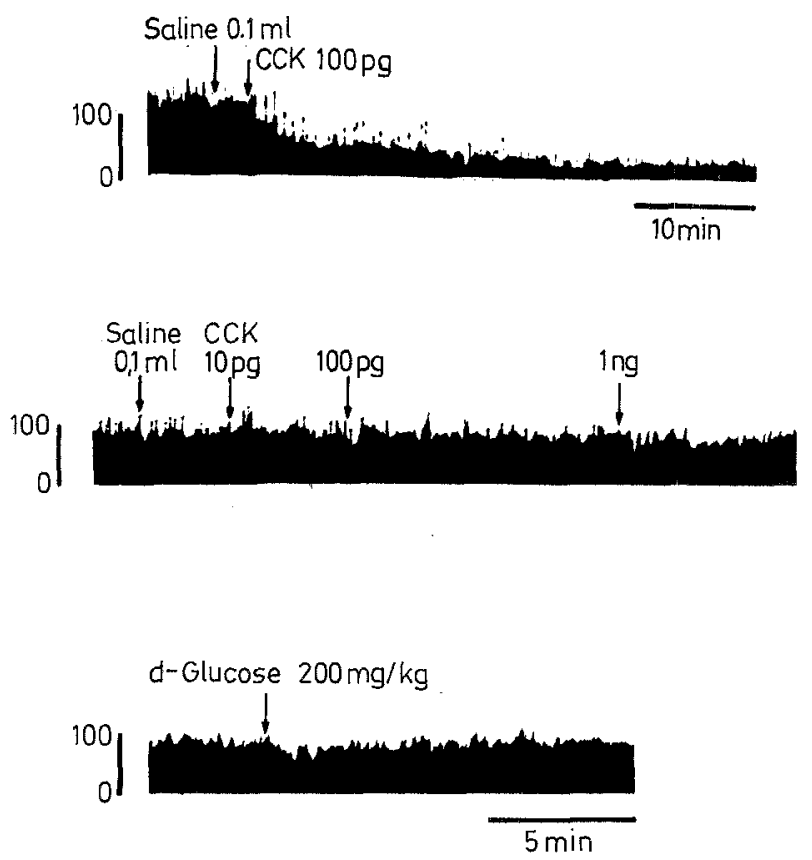

Fig. 1. Effect of CCK and glucose on the firing rate of vagal hepatic afferents (Rat). Infusions were made into the portal vein. Arrows show the time of the infusions. In the second trace, the infusion of CCK (10 pg) was without effect, $100 \mathrm{pg}$ of CCK caused a weak suppressive effect and $1 \mathrm{ng}$ caused a stronger and longer effect. Vertical bars show the rate of firing $(100 \mathrm{spikes} / 5 \mathrm{sec})$. Horizontal bars show the time

the administration, the firing rate still showed no recovery to the levels observed before administration. Figure 1 depicts the dose-response type suppressive effect of CCK and also the suppressive effect of glucose $(200 \mathrm{mg} / \mathrm{kg} ; 0,5 \mathrm{ml})$ injected into the portal vein on the firing rate of the same units.

As discussed earlier, injection of glucose into the portal vein suppresses food intake and reduces nerve signals from the liver to the central nervous system; suppression of food intake by CCK might be explained by a similar mechanism. Satiation after feeding due to the release of CCK from the gut to the portal vein can be explained in this way.

Effect of Serotonin ( $5 H T$ ). It was reported by Jaffe and other workers [6] that tissue concentrations of serotonin were highest in the duodenum. They also demonstrated that serotonin can be released from the duodenum into the portal circulation by acidification as well as by instillation of hypertonic glucose in the duodenum. Similar reports were made by other researchers $[5,7,8]$.

The following experiments showed the effect of serotonin on the firing rates of vagal hepatic afferents. Injections of serotonin (Wako Ltd.) dissolved in saline were made through a catheter inserted into the portal vein in the rat. As shown in Figure 2, an injection of serotonin ( $1 \mathrm{ng}, 0.1 \mathrm{ml}$ ) caused 

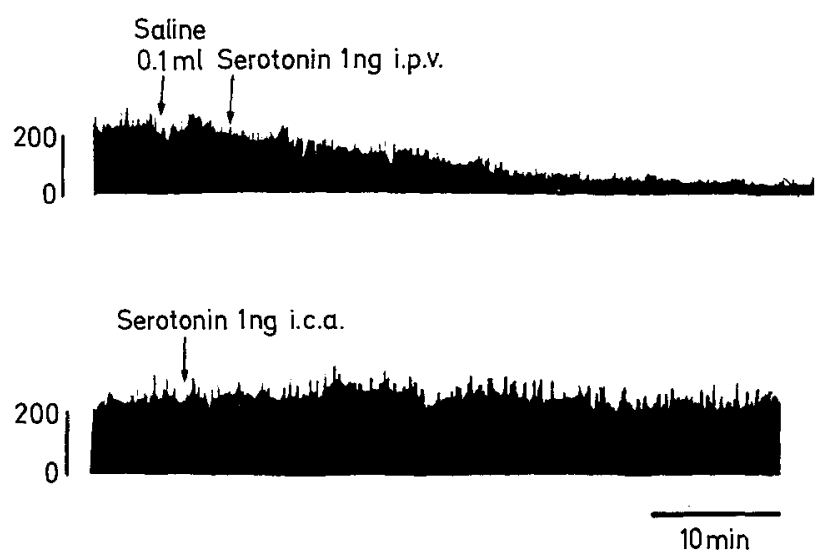

Fig. 2. Effect of serotonin on the firing rate of vagal hepatic afferents (upper trace) and vagal pancreatic afferents (lower trace) (Rat). Arrows show the time of infusions. Vertical bars show the rate of firing ( 200 spikes $/ 5 \mathrm{sec}$ ). Horizontal bars show the time

gradual and remarkable suppression of the afferent discharge rate. No recovery of the firing rates was observed by one hour after the administration. Serotonin liberated from the gut by means of adequate intraduodenal stimulation may cause similar depression of the firing rate and may in turn cause a decrease of food intake.

\section{Studies on Vagal Pancreatic Afferents}

Experiments were conducted in rabbits and rats. As shown in Figure 3, all mesenteric nerve branches and blood vessels from peripheral sites of the pancreas were tied to eliminate potential information arriving from the stomach, duodenum, intestine and spleen. After this procedure, the afferent discharges recorded from the peripheral cut end of the celiac branch of the vagus nerve were mainly from the pancreas. A catheter inserted into the cardiac side of the carotid artery or jugular vein was used for the administration of glucose, CCK, serotonin and other substances.

Effect of Glucose, 2 DG, Mannose and Insulin. As shown in Figure 4, the i.v. infusion of glucose $(150 \mathrm{mg} / \mathrm{kg})$ caused a decrease of the firing rate whereas an infusion of the same amount of mannose or Ringer's solution $(2 \mathrm{ml})$ was without effect. The i.v. administration of $2 \mathrm{DG}$ caused an increase in activity. The responses observed were similar to those observed in the glucose-sensitive vagal hepatic afferents.

An increase of the firing rate was also observed following the i.v. injection of a large amount of insulin (10 U) (Fig. 3). The effect of smaller doses of insulin was not clear. The effective dose of insulin seems to be too high to be a physiological stimulus to the afferent nerve terminals in the pancreas even though it is diluted by the blood in the systemic circulation. However, if the afferent nerve elements are situated close enough to the B-cells of the islets, high concentrations of insulin might normally bathe the nerve terminals. Similar effects of glucose and insulin were observed in rats.

Effect of CCK. An injection of CCK (100 ng, $0.1 \mathrm{ml}$ ) into the carotid artery caused a remakable increase of the discharge rate of the vagal pancreatic afferents. A carotid injection of CCK $(100 \mathrm{pg}, 0.1 \mathrm{ml})$ was without effect. The dose-response effect is depicted in Figure 5. This effect of CCK is opposite to that observed on the rate of firing in the vagal hepatic afferents. The latter showed a suppressive response and the former demonstrated an enhancement of discharge rate.

Effect of Serotonin. In 1957 Douglas and Ritchie [4] observed that an increase of activity of the cervical vagus came from the gastrointestinal tract following an i.v. infusion of serotonin. Recently, Niijima [16] reported that a carotid or intraduodenal infusion of serotonin (10-100 ng) caused an increase of the afferent discharge rate of the mesenteric nerve. Subsequent experiments showed the effect of a carotid infusion of serotonin on the afferent activity of the pancreatic branch of the vagus nerve in the rat. All the procedures for recording the vagal pancreatic afferents were the same as those in the experiments in the rabbit.

An intracarotid injection of serotonin (1 ng, $0.1 \mathrm{ml}$ ) caused an increase of the firing rate with a delay of about $10 \mathrm{~min}$ (Fig. 2). Repeated experiments yielded similar results.

\section{Conclusion}

The results of there experiments have shown that there are similar types of glucose-sensitive vagal afferent nerve fibers in the liver and the pancreas. These afferents have decreased activity following the administration of glucose and increased activity after the injection of $2 \mathrm{DG}$. These afferent elements may be a mechanism for responding to blood glucose similar to that of lateral hypothalamic neurons. The results also indicate that $\mathrm{CCK}$ and serotonin have the opposite effects on vagal hepatic afferents and on the pancreatic afferents. The decrease of the discharge rate of vagal hepatic afferents caused by CCK or serotonin may cause a suppression of food intake behavior. The increase of discharge rate in vagal pancreatic afferents resulting from CCK or serotonin or insulin suggests the existence of a neurol information system for these substances in the circulating blood to the pancreas. 


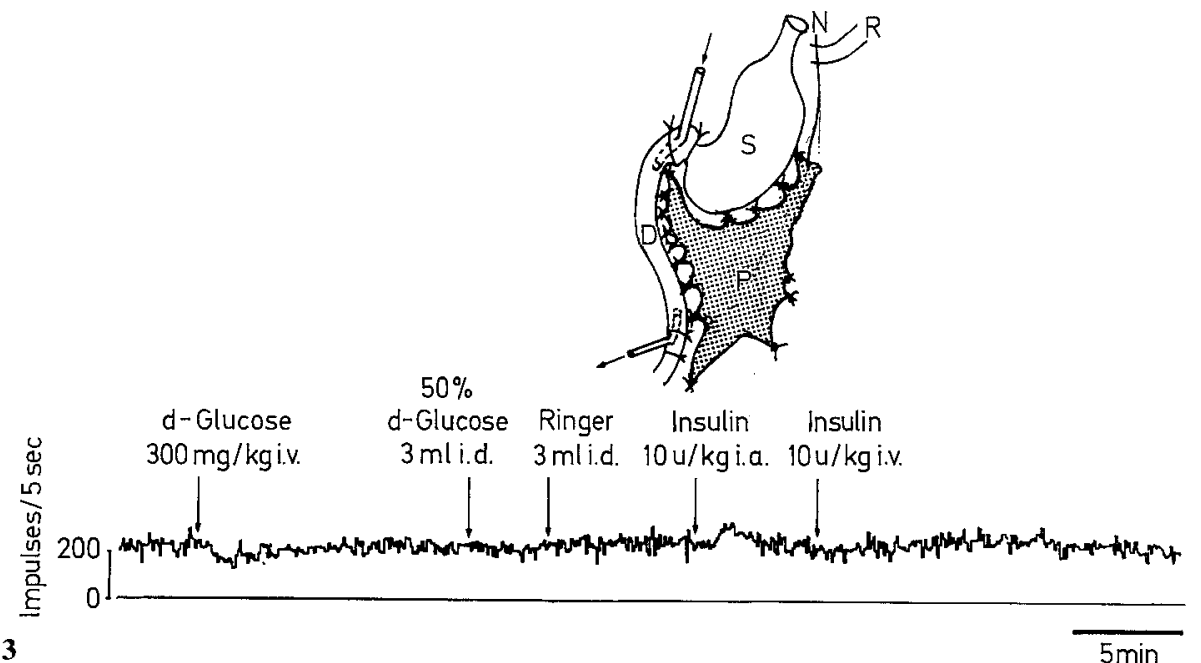

3

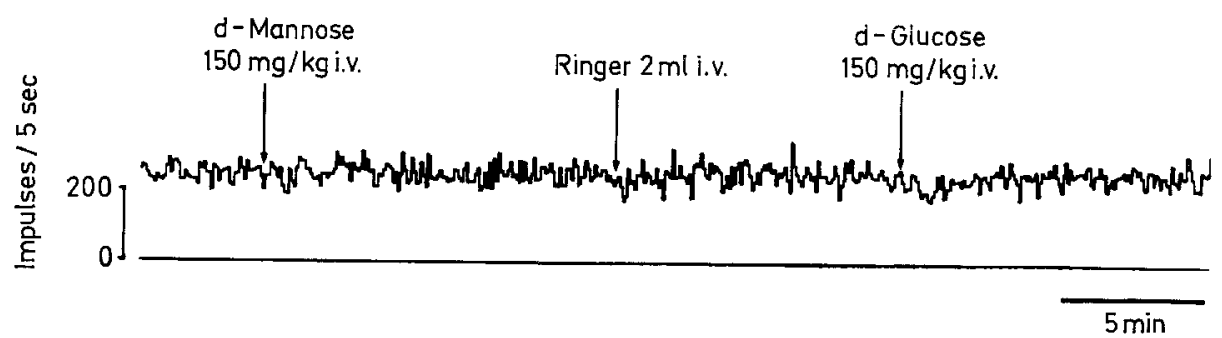

Fig. 3. Effect of glucose and insulin on the firing rate of vagal pancreatic afferents (Rabbits). Arrows show the time of infusion. P: Pancreas, S: Stomach, D: Duodenum, N: Vagal coeliac branch, R: Recording electrodes

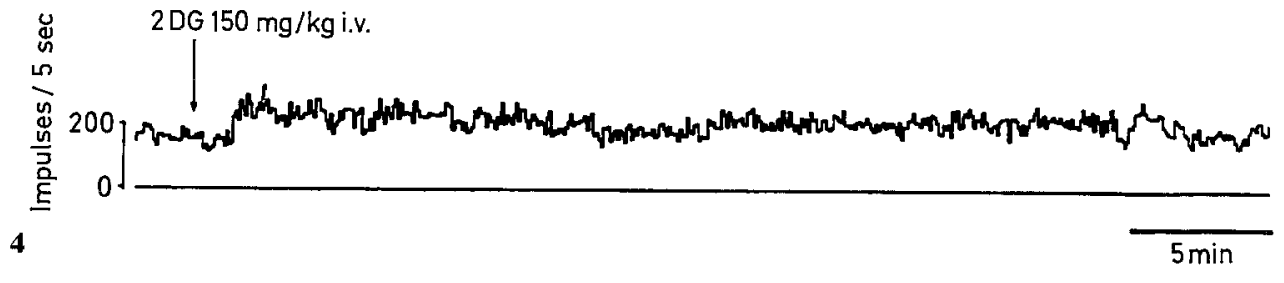

Fig. 4. Effect of mannose, glucose and 2 deoxyglucose on the firing rate of vagal pancreatic afferents (Rabbit). Arrows show the time of infusion

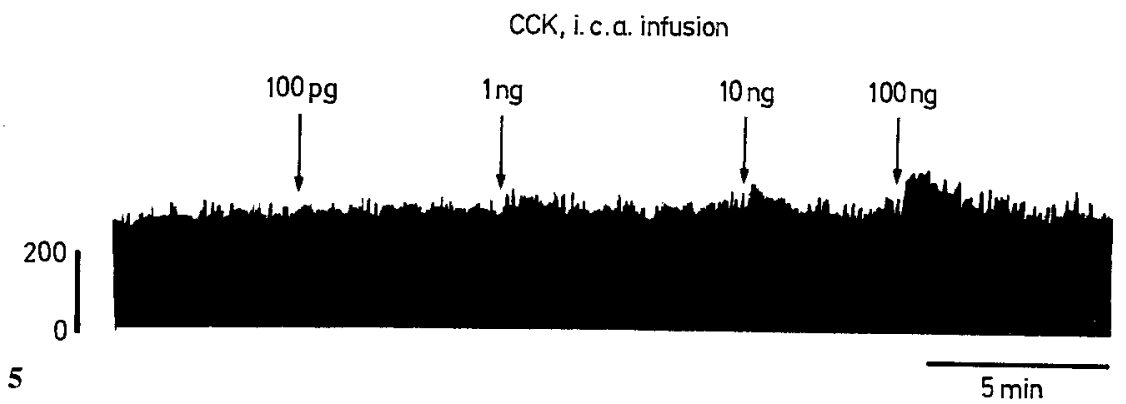

Fig. 5. Effect of Cholecystokinin on the firing rate of vagal pancreatic afferents (Rat). Arrows show the time of the infusion. i.c.a. stands for intra carotid arterial
Acknowledgements. The author would like to express his thanks to Prof. Beatrice M. Callery (Prof. of English, Niigata University, Japan) for her suggestions and advice on the manuscript, and to Prof. Stephen C. Woods for providing the CCK- 8 .

\section{References}

1. Anand BK, Chhina GS, Sharma K, Dua S, Singh B (1964) Activity of single neurons in the hypothalamic feeding centers: effect of glucose. Am J Physiol 207: 1146-1154

2. Antin J, Gibbs J, Holt J, Young RC, Smith GP (1975)
Cholecystokinin elecits the complete behavioral sequence of satiety in rats. J Comp Physiol Psychol 89: 784-900

3. Crovum WL (1977) Factors that decrease food intake by sheep. In: Abstracts of 6th Internat. Conf. on the Phyiol. of Food and Fluid Intake

4. Douglas WW, Ritchie JM (1957) On excitation of nonmedullated afferent fibers in the vagus and aortic nerves by pharmacological agents. J Physiol (Lond) 138: 31-43

5. Drapanas T, McDonald JC, Stewart JD (1962) Serotonin release following instillation of hypertonic glucose into the proximal intestine. Ann Surg 156: 528-536

6. Jaffe BM, Kellum Jr JM, Kopen DF, Stechenberg L (1978) 
Release and physiologic action of serotonin. In: Bloom SR (ed) Gut hormones. Churchhill Livingstone, Edinburgh London New York, p 515-523

7. Johnson LP, Jesseph JE (1961) Evidence for a humoral etiology of dumping syndrome. Surg Forum 12: 316

8. Johnson LP, Sloop RD, Jesseph JE, Harkins HN (1962) Serotonin antagonists in experimental and clinical "dumping". Ann Surg 56: 537-545

9. Gibbs J, Falasco JD, Maddison SP, Rolls ET (1977) The role of the intestine in satiety examined in sham-feeding rhesus monkeys. In: Abstracts of 6th Internat. Conf. on the Physiol of Food and Fluid Intake

10. Houpt RT, Antika SM (1977) Satiety of cholecyctokinin and caerulein in rabbits. In: Abstracts of 6th Internat. Conf. on the Physiol. of Food and Fluid Intake

11. Lorenz DW (1977) Evidence that the satiety effect of cholecystokinin is physiological, not pharmacological. In: Abstracts of 6th Internat. Conf. on the Physiol of Food and Fluid Intake

12. Mei N (1978) Vagal glucoreceptors in the small intestine of the cat. J Physiol (Lond) 282: 485-506

13. Niijima A (1969) Afferent impulse discharges from glucoreceptors in the liver of the guinea pig. Ann N Y Acad Sci 157: $690-700$

14. Niijima A (1979) Control of liver function and neuroendocrine regulation of blood glucose levels. In: Brooks C McC, Koizumi K, Sato A (eds) Integrative functions of the Autonomic nervous system. Univ of Tokyo Press, Tokyo; Elsevier/ North Holland/Biomed Press, Amsterdam p 68-83

15. Niijima A (1980) Glucose sensitive afferent nerve fibers in the liver and regulation of blood glucose. Brain Res Bull 5 [Suppl 4]: $175-179$

\section{Discussion after Niijima's Presentation}

Woods: Gerry Smith claims that CCK influences feeding by acting at receptors in the stomach (Soc Neurosci Abstr (1979) 5: 224) Have you looked at the effect of CCK upon gastric afferents?

Niijima: An IV injection of CCK-8 caused an increase of gastric afferent activity.

Fernstrom: Your hypothesis that both glucose and serotonin act in the same manner, perhaps even on the same fibers, to reduce feeding by decreasing hepatic afferent input to the CNS is very interesting. Perhaps your technique could be used to see if fenfluramine acts at the liver since it reduces food intake but is not known for certain to work exclusively at the CNS.

Samols: Are there afferent nerve endings within the small venules surrounding the islets?

Smith: There are no reports of this of which I am aware.

Berthoud: The cell bodies of the afferent vagal fibers from which you record are up near the brainstem such that when you cut the fibers, you must be killing them at the same time. Are your results meaningful?

Niijima: We feel that the fibers are alive and functioning well. When we perfuse with Ringer's solution and $95 \%$ oxygen, we have been able to record from the same fibers for up to 5 hours (Ann NY Acad Sci (1969) 157:600); and most of our experiments are much more acute. There is no change of the spontaneous firing rate over several hours.

Berthoud: Your experiment are always so elegant. I was
16. Niijima A (in press) Effect of serotonin (5 HT) on the firing rate of afferent discharges recorded from the mesenteric nerves in the rat. Biomed Res

17. Novin D (1977) The mechanisms of satiety. In: Katsuki Y, Sato M, Takagi SF, Oomura Y (eds) Food intake and chemical senses Univ of Tokyo Press, Tokyo, p 399-411

18. Oomura Y, Kimura K, Ooyama H, Maeo T, Iki M, Kuniyoshi $N$ (1954) Reciprocal activities of the ventromedial and lateral hypothalamus areas of cats. Science 143: 484-485

19. Oomura Y, Ooyama H, Yamamoto T, Ono T, Kobayashi N (1969) Behavior of hypothalamic unit activity during electrophoretic application of drugs. Ann NY Acad Sci 157: 642-665

20. Oomura $Y$, Ooyama H, Sugimori M, Nakamura T, Yamada $Y$ (1974) Glucose inhibition of glucose sensitive neurone in the rat lateral hypothalamus. Nature 247: 284-286

21. Russek M (1963) Parcipitation of hepatic glucoreceptors in the control of intake of food. Nature 197: 79-80

22. Russek M (1970) Demonstration of the influence of an hepatic glucose-sensitive mechanism on food intake. Physiol Behav 5: 1207-1209

23. Smith GP, Gibbs J, Young RC (1974) Cholecystokinin and intestinal satiety in the rat. Fed Proc 33: 1146

24. Sturdevant RAL, Goetz H (1976) Cholecystokinin both stimulates and inhibits human food intake. Nature 261: $713-715$

Akira Niijima

Department of Physiology

Niigata University School of Medicine

Niigata City 951

Japan

wondering if you have a special kind of recording electrode or if there is any secret to your technical success?

Niijima: We use a standard pair of silver wire recording electrodes and equipment. We do find the use of a binocular dissecting microscope very helpful in identifying the finer nerve fibers.

B. Jeanrenaud: I would like a little more clarification regarding your data and what is known about food intake. Have you ever looked at behavior?

Niijima: No, we haven't. Our data are indicative of signals originating in peripheral organs and passing to the CNS. We can only correlate our findings with the physiological experiments of other in which compounds such as glucose and CCK are administered peripherally and food intake is measured.

Novin: I would like to expand on that a little. Our behavioral data are very consistent with Dr. Niijima's electrical data. We have found a suppression of food intake when glucose was infused into either the liver of the gut, depending upon the nutritional state of the animal. Consistent with this, vagotomy eliminated or reduced the effect.

Smith: Have you ever measured insulin or glucagon to see what is happening in the pancreas when these neurons are activated.

Niijima: No, we have not done those experiments. Others have generated nerve impulses in the same nerves and inhilited insulin secretion, however (Brain Res (1979) 164: 314). 